\title{
Anticholinergic Toxicity Related to the Use of Datura Innoxia
}

\author{
Mustafa Baştürk1', Mustafa Içer², Cahfer Güloğlu², Selçuk Ertekin³, Serkan Doğan' \\ 'Department of Emergency Medicine, Kanuni Sultan Süleyman Training and Research Hospital, İstanbul, Turkey \\ 2Department of Emergency Medicine, Dicle University School of Medicine, Diyarbakır, Turkey \\ ${ }^{3}$ Department of Biology, Dicle University Science and Art Faculty, Diyarbakır, Turkey
}

\begin{abstract}
Introduction: The incorrect use of the flowers, leaves, and seeds of some plants by members of the general public can give rise to serious toxicities. Datura innoxia (DI), also known as pipe grass or weed wizard, is frequently used for the treatment of asthma and cough and to alleviate cramps. However, DI is also a hallucinogenic, with tremendous potential for abuse and can cause anticholinergic toxicity, and serious and fatal complications, such as coma, respiratory failure, and death, have been reported.

Case Report: We present the cases of two women (21 years old and 41 years old) who were admitted to the emergency department with findings of anticholinergic toxicity, including mydriasis, agitation, and delirium, after taking DI. The patients' pupils were widely dilated. Gastric decontamination and administration of activated charcoal, IV fluids, and midazolam/ diazepam were performed. The patients' general conditions were recovered except for mydriasis. However, after 1 week, the diameter of the patients' pupils returned to normal.

Conclusion: The unconscious misuse of some plants can cause fatal anticholinergic toxicity. Such toxicity though has rarely been reported with DI. When plants known to cause toxicity are usually endemic, clinicians should be alert to potential anticholinergic toxicity in patients who present with anticholinergic symptoms in these endemic areas.
\end{abstract}

Keywords: Anticholinergic toxicity, herbal therapy, Datura innoxia

Received: 09.03.2016 Accepted: 18.04.2016 Available Online Date: 15.07.2016

\section{Introduction}

The accidental or deliberate consumption of some plants and herbal products by individuals may lead to mortal intoxications (1). These plants, some of which have the potential for therapy, nutrition, and abuse, are listed in toxicity tables. Datura innoxia (DI), also known as downy thorn-apple, is a species in the Solanaceae family that is mostly used for asthma treatment and to palliate cramps. Moreover, it has hallucinogenic effects and this can cause a probability for abuse $(1,2)$. Furthermore, the plant contains L-hiyosiyamin, atropine, and scopolamine and can cause anticholinergic toxicity by the competitive inhibition of muscarinic cholinergic receptors (3). The incidence of mortal complications, such as respiratory insufficiency, coma, and death, is predicted to be more than $5 \%$, and it is known that the severity of complications depends on the level of anticholinergic toxicity (4).

Herein, we present the cases of two patients who were brought to the emergency service because of some signs and symptoms associated with possible anticholinergic intoxication. After DI was taken by the patients, mydriasis and excitability symptoms had occurred, indicating a rare cause of anticholinergic toxicity resulting from DI.

\section{Case Reports}

Case 1. A 41-year-old woman was admitted to the emergency service with excitation, delirium, and impaired general condition 2 hours after having breakfast. On the initial physical examination, the vital signs were as follows: blood pressure 140/90 mmHg, heart rate $140 / \mathrm{min}$, and respiratory rate $24 / \mathrm{min}$. The patient was agitated and aggressive, with abnormal involuntary movements and delirium. The patient's pupils were widely dilated and not reactive to light. The results of laboratory studies were all 
within the normal range. The family reported that the patient had suffered from asthma for 15 years and she had drunk a beverage containing DI during the previous 4 days. After admission, gastric decontamination was provided, owing to gastric lavage, together with activated charcoal. Intravenous fluids and midazolam (2 mg) were administered. The patient remained agitated, and an infusion of midazolam was started with doses of $0.15 \mathrm{mg} / \mathrm{kg} / \mathrm{h}$ during the first $4 \mathrm{~h}$ after hospitalization. On the $12^{\text {th }}$ hour of hospitalization, a repeated neurological examination revealed a gradual improvement in the patient's state of consciousness, with the disappearance of agitation and delirium. The clinical course was favorable. On the third day of hospitalization, the patient was discharged. The patient was followed up on a daily basis because of a lack of improvement in the mydriasis. However, by the seventh day, the diameter of the patient's pupils had returned to normal. Written informed consent was obtained from the patient for publishing this case report.

Case 2. A 21-year-old female was taken to the emergency room because of vomiting, disorientation, hallucinations, and impaired general condition $2 \mathrm{~h}$ after her lunch. On the initial physical examination, the vital signs were as follows: blood pressure 140/90 mmHg, pulse rate $82 / \mathrm{min}$, and respiratory rate $22 / \mathrm{min}$. The patient was agitated and aggressive with purposeless movements, and the patient's pupils were widely dilated. The results of laboratory studies were all within the normal range. The family reported that the patient had eaten some food that contained DI. Immediately after admission, a gastric tube was inserted to perform nasogastric lavage and administer activated charcoal. Intravenous fluids and diazepam (5 mg) were administered. On the $12^{\text {th }}$ hour of hospitalization, a repeated neurological examination revealed a gradual improvement in the patient's state of consciousness with the disappearance of agitation and delirium. The clinical course was favorable. On the third day of hospitalization, the patient was discharged. The patient was followed up on a daily basis because of a lack of improvement in mydriasis. However, by the fifth day, the diameter of the patient's pupils had returned to normal. Written informed consent was obtained from the patient for publishing this case report.

\section{Discussion}

Although the prevalence of anticholinergic toxicity due to drugs such as antidepressants, phenothiazines, antihistamines, and antiParkinson agents is high, anticholinergic toxicity dependent on plants is rare (5).

The relatives of the patients in the two presented cases brought the plant to the hospital. An expert from the department of biology at the university was called up and thus it was confirmed that the plant was DI (Figure 1). DI is an annual shrubbery plant, which blooms in June-November and is native to Central America. DI can be found in Mediterranean woodlands. DI typically reaches a length of 120-180 cm. The stems and leaves are covered with downy hairs. The flowers are white and their shape resembles a trumpet. Additionally, their length is approximately $15-17 \mathrm{~cm}$, and they consist of five segments. The flower buds are erect in an early phase, but they are inclined to droop downwards after some time. Droop is caused

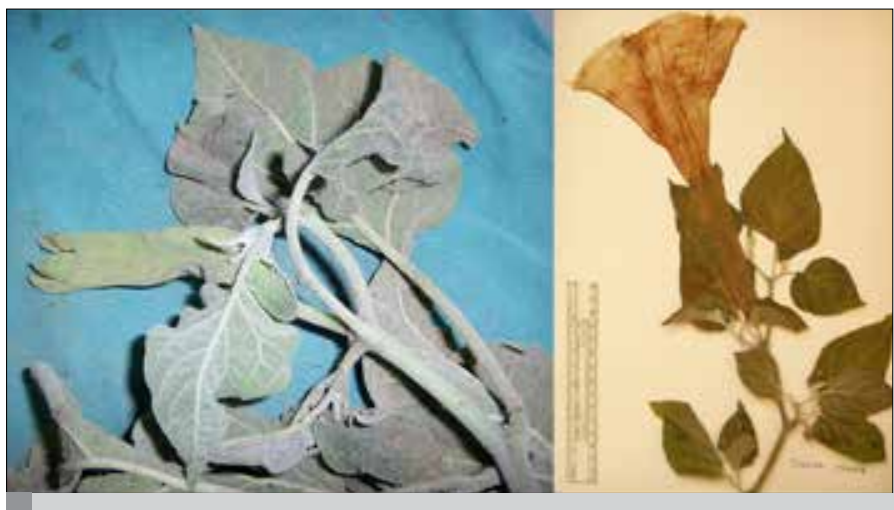

FIGURE 1. a, b. The plants of Datura İnnoxia (a) fresh leaf (b) dry leaf

by the seed head, covered with myriad thorns. The plant produces a thorny fruit (6). Intoxication may occur following ingestion of the seeds, flowers, or leaves of this plant. Overall, 100 seeds of Datura species contain approximately $6 \mathrm{mg}$ of atropine and alkaloids. Oral intake of this amount can be fatal (7).

Anticholinergic intoxication leads to central nervous system changes, such as agitation, amnesia, anxiety, ataxia, coma, confusion, delirium, hallucinations, hyperactivity, lethargy, seizures, respiratory failure, and mydriasis, as a result of central cholinergic blockade. It also produces peripheral effects, such as arrhythmia, tachycardia, reduced bronchial secretions, red skin, hypo-hypertension, hyperthermia, dry skin, mucous membranes, and urinary retention, due to peripheral cholinergic blockade $(5,8)$. Symptoms can occur within 30-60 min of ingesting the plants. However, the onset of symptoms may take up to $48 \mathrm{~h}$ as a result of a delay in gastrointestinal tract motility (9). Patients presenting with these symptoms should alert physicians for the suspicion of anticholinergic intoxication. In our patients, mydriasis, agitation, and delirium were the presenting symptoms.

The treatment of patients with anticholinergic toxicity includes gastric decontamination with nasogastric lavage, activated charcoal, and support therapy. Because of the possibility of prolonged symptoms, decontamination should be performed within the first $48 \mathrm{~h}$ of a patient being admitted. Benzodiazepines can be used to control seizures and agitation. Physostigmine can be used in cases of unstable tachycardia, ineffectiveness of support treatment, seizures that do not respond to benzodiazepines, uncontrollable agitation, coma with respiratory depression, and malignant hypertension. Our patients' complaints began $2 \mathrm{~h}$ after ingestion of the DI plant. Intravenous benzodiazepine was administered to control their agitation and delirium. However, gastric decontamination was performed with nasogastric lavage and activated charcoal. Twelve hours after admission to the hospital, a neurological examination revealed that agitation and delirium had resolved. In these patients, the most persistent symptoms were blurred vision and mydriasis. These symptoms may continue for approximately 1 week (5). Mydriasis recovered by the fifth and seventh day for case 1 and case 2 , respectively. Moreover, no complications were observed in our patients. 


\section{Conclusion}

Consequently, the unconscious consumption of some herbal products may cause mortal anticholinergic intoxication. Toxicity related to DI is not common. If plants associated with intoxication are present at the scene of an accident, this must alert clinicians in terms of a possible anticholinergic intoxication in patients who have relevant signs and symptoms.

Informed Consent: Written informed consent was obtained from patient who participated in this case.

Peer-review: Externally peer-reviewed.

Author contributions: Concept - M.B., M.I., C.G.; Design - M.B., S.D.; Supervision - M.B., M.I.; Resource - M.B., S.E.; Materials - M.B., M.I.; Data Collection\&/ or Processing - M.B., M.I.; Analysis\&/or Interpretation - M.B., S.D.; Literature Search - M.B., S.D.; Writing - M.B.; Critical Reviews - M.B., S.D.

Conflict of Interest: The authors declared no conflict of interest.

Financial Disclosure: The authors declared that this study has received no financial support.

\section{References}

1. Guven H. Environmental Toxins: Herbal Medicines and Poisonings. Turkiye Klinikleri J Pharmacol-Special Topics 2003; 1: 58-61.

2. Celbek G, Gunes H, Gungor A, Cecen F, Basar C, Sarıtas A, et al. Anticholinergic syndrome after trial of datura stramonium: A Case Report. Konuralp Medical Journal 2010; 2: 35-7.

3. Raman SV, Jacob J. Mydriasis due to datura inoxia. Emerg Med J 2005; 22: 310-1. [CrossRef]

4. Marc B, Martis A, Moreau C, Arlie G, Kintz P, Leclerc J. Acute datura stramonium poisoning in an emergency department. Presse Med 2007; 36: 1399-1403. [CrossRef]

5. Wolf L. Anticholinergic toxicity Tintinalli J, Kelen G, Stapcznski JS (eds). Emergency medicine: a comprehensive study guide. New york: mcgraw-hill; 1999, pp 1182-1184.

6. http://www.flowersinisrael.com/daturainnoxia_page.htm.

7. Salen P, Shih R, Sierzenski P, Reed J. Effect of physostigmine and gastric lavage in a Datura stramonium-induced anticholinergic poisoning epidemic. Am J Emerg Med 2003; 21: 316-7. [CrossRef]

8. Kemmener DA. Anticholinergic syndrome. J Emerg Nurs 2007; 33: 76-8. [CrossRef]

9. www.cdc.gov/mmwr/PDF/wk/mm4403.pdf Centers for disease control and prevention (cdc). Morbidity and mortality weekly report. Jimson weed poisoning--texas, New York, and California, 1994. MMWR Morb Mortal Wkly Rep 1995; 44: 41-4. 